\title{
Erratum to: Overweight/obesity and underweight are both risk factors for osteoporotic fractures at different sites in Japanese postmenopausal women
}

\author{
S. Tanaka $\cdot$ T. Kuroda $\cdot$ M. Saito $・$ M. Shiraki
}

Published online: 23 January 2013

(C) International Osteoporosis Foundation and National Osteoporosis Foundation 2013

Erratum to: Osteoporos Int

DOI 10.1007/s00198-012-2209-1

The authors mistakenly reported incorrect mean values and SDs for 1,25-dihydroxyvitamin D in the last row of Table 1. The correct means (SDs) are 19.3 (6.2) for underweight, 20.1 (6.0) for normal weight, and 20.4 (6.1) for overweight/obesity.

The online version of the original article can be found at http:// dx.doi.org/10.1007/s00198-012-2209-1.

S. Tanaka $(\bowtie)$

Division of Clinical Trial Design and Management,

Translational Research Center, Kyoto University,

54 Shogoin Kawahara-Cho, Sakyo-ku,

Kyoto 606-8507, Japan

e-mail: shiro@kuhp.kyoto-u.ac.jp

T. Kuroda

Public Health Research Foundation, Tokyo, Japan

M. Saito

Department of Orthopaedic Surgery, Jikei

University School of Medicine, Tokyo, Japan

M. Shiraki

Research Institute and Practice for Involutional Diseases,

Nagano, Japan 
Table 1 Baseline characteristics of the 1,614 postmenopausal women according to body mass index

\begin{tabular}{|c|c|c|c|c|c|c|c|}
\hline & \multicolumn{2}{|c|}{ Underweight $(N=135)^{\mathrm{b}}$} & \multicolumn{2}{|c|}{ Normal weight $(N=1,131)$} & \multicolumn{2}{|c|}{ Overweight/obese $(N=348)^{\mathrm{b}}$} & \multirow[t]{2}{*}{$p^{\mathrm{c}}$} \\
\hline & Mean & SD & Mean & SD & Mean & SD & \\
\hline Age (year) & 65.5 & 14.3 & 62.5 & 11.2 & 63.2 & 10.1 & - \\
\hline BMI $\left(\mathrm{kg} / \mathrm{m}^{2}\right)$ & 17.2 & 1.2 & 21.9 & 1.7 & 27.2 & 2.4 & - \\
\hline Weight $(\mathrm{kg})$ & 39.4 & 4.8 & 50.4 & 5.8 & 61.4 & 7.8 & $<0.01$ \\
\hline Lean mass $(\mathrm{kg})$ & 31.6 & 3.2 & 34.1 & 3.4 & 36.1 & 3.5 & $<0.01$ \\
\hline Fat mass $(\%)$ & 19.8 & 6.5 & 31.4 & 5.8 & 40.0 & 4.6 & $<0.01$ \\
\hline Waist circumference $(\mathrm{cm})$ & 74.8 & 7.7 & 83.9 & 7.5 & 93.0 & 10.5 & $<0.01$ \\
\hline DM (\%) & $3.7 \%$ & & $6.1 \%$ & & $16.1 \%$ & & $<0.01$ \\
\hline Hypertension (\%) & $58.5 \%$ & & $66.0 \%$ & & $79.9 \%$ & & $<0.01$ \\
\hline Hyperlipidemia (\%) & $30.4 \%$ & & $50.5 \%$ & & $64.4 \%$ & & $<0.01$ \\
\hline Smoker $(\%)$ & $2.3 \%$ & & $2.6 \%$ & & $3.8 \%$ & & 0.17 \\
\hline Treated by conjugated estrogen or estradiol & $7.4 \%$ & & $6.9 \%$ & & $2.9 \%$ & & 0.01 \\
\hline eGFR $\left(\mathrm{mL} / \mathrm{min} / 1.73 \mathrm{~m}^{2}\right)$ & 62.2 & 19.5 & 63.9 & 20.2 & 66.4 & 62.2 & 0.04 \\
\hline Osteoporosis $(\%)^{\mathrm{a}}$ & $57.8 \%$ & & $31.3 \%$ & & $21.0 / 15.3 \%$ & & $<0.01$ \\
\hline Osteopenia $(\%)^{\mathrm{a}}$ & $19.3 \%$ & & $22.1 \%$ & & $21.0 / 15.3 \%$ & & 0.06 \\
\hline Prior fracture $(\%)$ & $23.7 \%$ & $42.7 \%$ & $17.4 \%$ & $37.9 \%$ & $15.8 \%$ & $23.7 \%$ & 0.65 \\
\hline Lumbar BMD $\left(\mathrm{g} / \mathrm{cm}^{2}\right)$ & 0.821 & 0.220 & 0.955 & 0.197 & 1.037 & $0.199 / 0.144$ & $<0.01$ \\
\hline Femur BMD $\left(\mathrm{g} / \mathrm{cm}^{2}\right)$ & 0.661 & 0.121 & 0.774 & 0.131 & 0.844 & $0.199 / 0.144$ & $<0.01$ \\
\hline Back pain $(\%)$ & $34.1 \%$ & & $29.3 \%$ & & $26.4 \%$ & & 0.19 \\
\hline BAP (IU) & 30.8 & 10.9 & 30.6 & 11.8 & 31.4 & 11.4 & 0.45 \\
\hline NTX (nM/mMCr) & 56.0 & 29.8 & 51.3 & 27.2 & 50.3 & 26.9 & 0.20 \\
\hline Osteocalcin $(\mathrm{ng} / \mathrm{mL})$ & 8.6 & 4.2 & 7.8 & 3.5 & 7.4 & 7.2 & 0.02 \\
\hline ucOC (ng/mL) & 5.2 & 2.4 & 4.6 & 3.1 & 4.7 & 3.2 & 0.87 \\
\hline $25(\mathrm{OH}) \mathrm{D}(\mathrm{ng} / \mathrm{mL})$ & 19.3 & 6.2 & 20.1 & 6.0 & 20.4 & 6.1 & 0.23 \\
\hline
\end{tabular}

$S D$ standard deviation, $B M I$ body mass index, $D M$ diabetes mellitus, $e G F R$ estimated glomerular filtration rate, $B M D$ bone mineral density, $B A P$ bone alkaline phosphatase, $N T X \mathrm{~N}$-terminal telopeptide, ucOC undercarboxylated osteocalcin, 25(OH)D 25-hydroxy-cholecalciferol

${ }^{a}$ Osteoporosis is defined as lumbar BMD < YAM80 \% (-1.63 SD) with fragility fractures or lumbar BMD < YAM70 \% ( 2.45 SD) without fragility fractures. Osteopenia is defined as lumbar BMD $<$ YAM $80 \%(-1.63 \mathrm{SD})$ without osteoporosis

${ }^{\mathrm{b}}$ Underweight, overweight, and obesity are defined by a BMI of less than $18.5 \mathrm{~kg} / \mathrm{m}^{2}$, between 25 and $29 \mathrm{~kg} / \mathrm{m}^{2}$, or $30 \mathrm{~kg} / \mathrm{m}^{2}$ or more, respectively

${ }^{\mathrm{c}}$ Trend test adjusted for age 